\title{
Le cosmos comme terrain de jeu : l'espace dans l'animation soviétique et russe
}

\author{
Birgit Beumers \\ Université d'Aberystwyth
}

Nina Sputnits Kaya

Institut de recherches scientifiques de l'Institut national russe de cinema

(VGIK)

Traduit par Hélène MÉLAT

\section{Introduction}

Les étoiles et les planètes ont toujours intéressé le cinéma depuis sa création. Alors que les frères Lumière capturaient des scènes terrestres, comme des trains arrivant sur les quais (L'Arrivée d'un train à La Ciotat, 1895) et des ouvriers quittant l'usine (La Sortie de l'usine Lumière à Lyon, 1895), Georges Méliès, le cinéaste magicien amateur de trucages, créait les premiers vaisseaux spatiaux jamais imaginés et inventait des voyages imaginaires vers d'autres planètes : La Lune à un mètre (1898) décrit la lune sous un jour fantastique, la montrant à la fois comme un cercle avec un visage et comme une faucille. Son Voyage dans la lune (1902), plus sophistiqué et colorié à la main, montre un groupe d'astronomes voyageant vers la lune, d'où ils s'enfuient après avoir rencontré des habitants terrifiants. Bien que ces premières expériences de représentation d'autres planètes n'aient pas été animées, elles ont tout de même utilisé des trucages et des effets spéciaux qui requièrent des savoir-faire et des technologies typiques de l'animation: la technique de l'image par image et les images composites. 
SLOVO

184 À l'Est de Pixar : le film d'animation russe et soviétique - no 48/49

Ce type d'effets spéciaux a été développé dans les films en prises de vues réelles soviétiques (y compris de science-fiction) des années 1920 et 1930, avec les possibilités qu'offre le set design pour le décor des autres planètes et les techniques d'animation pour la représentation du cosmos. Cet intérêt a coïncidé avec l'industrialisation rapide des années 1920, qui a mis au premier plan les processus mécaniques et la rationalisation du travail (avec le taylorisme) tout en prenant part à la création imaginaire romantique d'autres mondes: «Dans les années 1920, la technologie a joué un rôle primordial dans la production, la discussion et la facilitation sociales d'utopies ${ }^{1} \gg$. Le film Aelita [Aèlita] (1924) de Iakov Protazanov combine ces stratégies narratives et technologiques: il est centré sur le voyage imaginaire sur la planète Mars de l'ingénieur Los, qui apporte la révolution dans l'espace et reflète ainsi la politique soviétique de l'époque, qui consistait à envoyer des messages révolutionnaires dans les autres pays. Les décors constructivistes d'Aleksandra Ekster pour la vie sur Mars reflètent les intérêts esthétiques de l'époque, combinant technologie et vision artistique. La conquête de la planète Mars reste cependant cantonnée au rêve de Los. Comme dans les premières expériences cinématographiques, la vie sur d'autres planètes relève du domaine des contes de fées, comme le suggère Asif Siqqidi lorsqu'il lie élément imaginaire et vol spatial :

Premièrement, le vol spatial, qui était aussi lié à l'action de se libérer de la terre, poussait les limites physiques de l'émancipation au-delà du concevable, au-delà des frontières des cieux visibles. Deuxièmement, le vol spatial était un discours relevant entièrement de la fantaisie : les voyages au-delà de l'atmosphère n'avaient pas de précédents ni de modèle ${ }^{2}$.

Le vol spatial était un rêve qui allait devenir une réalité tangible - de même que l'utopie communiste qui attendait les citoyens soviétiques au bout de la voie révolutionnaire. Les deux se rencontrèrent sur le territoire des contes merveilleux, qui requièrent un espace parfait, fantastique, qui peut être inventé et créé grâce aux effets spéciaux et à l'animation.

1.SIDDIQI, 2008, p. 263.

2. Ibid., p. 264. 


\section{La colonisation de l'espace}

La combinaison de la technologie et d'un univers de rêve est liée au souhait d'apporter la révolution dans de nouveaux espaces et de nouvelles planètes. C'est ce qu'on trouve au cœur de La Révolution interplanétaire [Mežplanetnaja revoljucija] (1924), un film d'animation de Nikolaï Khodataev, Zenon Komissarenko et Iouri Merkoulov, qui devait initialement être inclus dans Aelita. Dans ce film, les bolchéviques partent exporter la révolution sur Mars, où les capitalistes du monde se sont réfugiés, mais la planète est déjà aux prises avec la révolution. Tout en étant une parodie visuelle d'Aelita, le film $s$ 'inscrit thématiquement dans la tradition de la confrontation globale opposant le capitalisme au communisme de la façon typique dont le font les cartoons de la fin des années 1920. La Révolution interplanétaire utilise pour déplacer les objets d'une image à l'autre une nouvelle technique très efficace, celle du papier découpé [perekladka], qui permet de rendre le mouvement entre les planètes. Les techniques et dessins de l'animation servent donc à projeter le monde réel dans l'espace, où le combat entre différents systèmes politiques se poursuit, anticipant dans un sens la course à l'espace pendant la Guerre froide.

Dans la mesure où le réalisme s'est imposé dans le discours culturel soviétique, culminant avec le réalisme socialiste, défini en 1934 comme la seule méthode pour la création artistique, l'espace était montré de manière très réaliste: les planètes devenaient des colonies du communisme et des clones de la terre, mais en plus parfait. Le voyage lui-même était plus important que la conquête, et l'animation et les effets spéciaux contribuaient à créer ce voyage dans l'espace cosmique de films en prises de vues réelles. Les animateurs et expérimentateurs des années 1930 participaient souvent à des films en prises de vues réelles, mais avec l'introduction du cellulo au milieu des années 1930, les technologies évoluèrent suffisamment pour que l'on puisse créer des mouvements fluides de divers objets dans des narrations autonomes, et le genre de l'animation devint florissant. À la fin des années 1930 et sous la direction du studio Soïouzmoultfilm nouvellement créé, l'animation se concentra sur les contes folkloriques, qui offraient, avec leur narration linéaire, l'intrigue idéale pour le mode narratif réaliste socialiste et pouvaient être aisément illustrés avec des images inspirées du folklore, suivant ainsi la phrase-clé de la politique des nationalités : « nationaliste dans la forme, socialiste dans le contenu ».

L'espace continue à être en vedette dans les films en prises de vues réelles des années 1930, où les effets spéciaux lui confèrent de la crédibilité; ces derniers sont créés par deux pionniers de la réalisation : Aleksandr Ptouchko (1900-1973) et Pavel Klouchantsev (1910-1999). Le projet non réalisé de Ptouchko racontant 
SLOVO

186 À l'Est de Pixar : le film d'animation russe et soviétique - no 48/49

un voyage sur Vénus, L'Étoile bleue [Golubaja zvezda] (1935), connu par la suite sous le titre L'Étoile du matin [Utrennjaja zvezda] (1939), comportait un ensemble sophistiqué d'effets spéciaux pour représenter le voyage spatial ${ }^{3}$. Et Le Vol spatial [Kosmičeskij rejs] (1935) de Vassili Jouravlev a bénéficié de l'excellente technique de l'opérateur, Aleksandr Galperine, et surtout du talent de Iouri Chvets, qui sera par la suite le dessinateur des productions de Klouchantsev et réalisera dans l'après-guerre les représentations novatrices du lancement d'une fusée et de l'apesanteur, comme Aurore boréale [Severnoe sijanie] (1946), Les Météores [Meteority] (1948) et La Voie vers les étoiles [Doroga $k$ zvëzdam] (1957). Klouchantsev a été le premier réalisateur à montrer de manière convaincante une pluie de météorites, l'aurore boréale, les planètes et les étoiles en orbite, et la surface des autres planètes. Il y est arrivé en combinant l'animation et les prises de vues réelles, les prises inversées, le filmage sous-marin et d'autres astuces de caméra. Certains de ces effets ont été copiés du cinéma américain ${ }^{4}$. Klouchantsev a réalisé une représentation précise et réaliste de l'espace sur écran et a construit des mondes de rêve techniquement impeccables, utilisant le cinéma pour créer des utopies socialistes du futur.

Nous avons osé filmer non pas ce qui existe, mais ce qui n'est pas encore là mais sera là bientôt, dans le futur. Nous avons osé non pas énoncer des faits, mais donner un pronostic, et nous avons fait cela non pas simplement par pure imagination mais avec des calculs fondés scientifiquement. Nous avons donc créé des objets qui n'existent pas encore. Nous avons inventé de nouvelles méthodes pour montrer l'environnement futur de l'homme. Nous avons conçu et construit de nouvelles technologies pour faire ces prises de vue. Tout cela n'entrait pas dans la base technique traditionnelle des films de vulgarisation; cela n'était pas adapté aux procédures de notre studio et de notre cinéma en général. Nous étions donc une gêne terrible dans la vie «normale » du studio. Nous nous mettions dans le chemin de tout le monde; nous irritions nos collègues et compliquions la gestion de la production ${ }^{5}$.

Les images utopiques de la vie humaine sur d'autres planètes sont aussi illustrées dans le film en prises de vues réelles de Klouchantsev La Planète des tempêtes [Planeta bur'] (1961), ainsi que plus tard dans les films de vulgarisation

3. Cf. Sputnitskaia, 2017.

4. Cf. Beumers, 2017, pp. 169-188.

5. KLUŠANCEV, 1997, p. 79. 
comme La Lune [Luna] (1965) et Mars [Mars] (1968), qui présentent la vie sur d'autres planètes sous la forme d'une version domestique de la vie sur terre. Dans Mars, la station spatiale est habitée par des familles avec des enfants, des animaux domestiques et des plantes. L'utopie soviétique de la vie parfaite a été projetée et étendue aux autres planètes, néanmoins cet idéal communiste ressemble aux salons des grand-mères du cinéma pré-révolutionnaire et du cinéma soviétique des débuts. Dans toutes ses entreprises, Klouchantsev a négligé le lien entre les autres planètes et la réalité contemporaine, lien qui était crucial pour fournir l'élément réaliste et connecter l'espace avec l'utopie communiste.

Grâce aux innovations techniques, il y a donc un glissement de l'exploration de l'espace à la colonisation d'autres planètes, qui deviennent des répliques en miniature des univers bourgeois domestiques ou l'équivalent de l'utopie socialiste au temps du « socialisme développé ». La colonisation n'a pas grand-chose à voir ici avec l'établissement d'un régime politique : on utilise plutôt les planètes pour installer des humains et trouver une harmonie qui apparemment - avec la course à l'espace et ses effets négatifs sur les relations soviéto-américaines - est impossible sur cette planète. Dans l'espace, les humanoïdes sont gentils et serviables, les voyages spatiaux ont en premier lieu et essentiellement une fonction éducative. De fait, pendant la guerre froide, l'Union soviétique a accéléré son programme spatial - le cosmodrome de Baikonour au Kazakhstan a été inauguré en février 1955. Le premier satellite Spoutnik a fait le tour de la terre en 1957, suivi par la capsule avec la chienne Laika. La sonde spatiale lunaire Luna 1 a été lancée en 1959, et en avril 1961, Iouri Gagarine a été le premier homme dans l'espace. Puis il y a eu la première femme dans l'espace, Valentina Terechkova, les premiers pas dans l'espace, réalisés par Alekseï Leonov en 1965. La conquête soviétique de l'espace était triomphante jusqu'à ce que les Américains mettent le pied sur la lune en 1969.

Dans le même temps, l'animation a pu développer une représentation autonome de l'espace, non pas en projetant des images mais en définissant la relation entre la terre et les autres planètes, entre le centre soviétique et ses satellites. La notion de colonisation demeure mais passe au second plan des films, qui sont essentiellement destinés aux enfants. L'évolution de l'espace dans l'animation est particulièrement intéressante pendant la période de la stagnation, car elle reflète le moment où l'animation est utilisée pour des effets spéciaux destinés à représenter l'espace de façon autonome, ce qui coïncide avec une autre évolution : la lune est conquise, et la course à l'espace passe de l'exploration à la conquête, les autres planètes deviennent un espace de vie alternatif pour des familles avec enfants et animaux, ce qui constitue un thème pour le cinéma d'animation pour enfants (ou, tout au moins, une version plus naïve de film de vulgarisation). Pour l'animation soviétique, l'espace est destiné aux familles, chiens et plantes inclus, avec et pour les enfants. 
SLOVO

188 À l'Est de Pixar : le film d'animation russe et soviétique - no 48/49

Le film de Valentina et Zinaïda Brumberg Volpour la lune [Polët na lunu] (1953) traite de la fascination de l'enfant pour le voyage spatial. Les protagonistes représentent le pays soviétique multi-ethnique - un Russe, un Ukrainien et un Noir de l'«Association internationale pour la communication interplanétaire » [Meždunarodnaja Organizacija Mežplanetnyx Soobšcenij, MOMS]. Ils observent la lune au télescope et suivent les rapports sur le premier vol humain vers la lune avec à son bord le père d'une petite voisine, Natacha. Les garçons harcèlent le professeur Bobrov, l'académicien en charge du programme lunaire, et apprennent que la fusée $\mathrm{R} 1$ a échoué sur la lune; R2 se prépare à décoller pour venir à la rescousse de R1, et Natacha ainsi que les garçons essaient d'introduire clandestinement le chien Tobik à bord de R2. Cependant, au lieu du chien, c'est l'un des garçons, Kolia, qui se retrouve à bord accidentellement. Une fois que la fusée est lancée, il est découvert. L'intérieur du vaisseau spatial ressemble à un salon, même si les tasses et soucoupes volent en accord avec les lois de la gravité. Tandis que Kolia s'inquiète à propos de ses examens à l'école, sa présence à bord de la fusée est évoquée dans les journaux, et un policier peut donner des nouvelles de Kolia aux autres garçons. Dans le programme spatial soviétique, rien n'est laissé au hasard, et même les circonstances les plus compliquées sont gérées de la manière la plus quotidienne qui soit. Ainsi, un concert de Tchaïkovski est transmis spécialement pour les cosmonautes (l'un d'entre eux est perdu sur la lune) : la vie soviétique est organisée et ordonnée, sur terre et dans l'espace. La fusée $\mathrm{R} 1$ a atterri dans un cratère et a perdu du fuel, ce qui est réparé quand $\mathrm{R} 2$ arrive, et finalement les deux vaisseaux spatiaux retournent sur terre. Kolia est très utile car il aide le professeur blessé à trouver le vaisseau spatial échoué. Le film montre l'espace avec des étoiles et des météorites, d'une manière assez grossière par rapport aux films scientifiques de Klouchantsev de la même époque, mais qui est adaptée aux enfants. Curieusement, les animaux ne sont pas admis dans l'espace, alors que les enfants le sont - mais avec une mission sérieuse à accomplir, ce n'est pas un jeu. Le film, tourné avant le premier lancement d'un satellite dans l'espace et avant que le voyage vers la lune ne soit devenu un but de la guerre à l'espace de la guerre froide entre les USA et l'URSS, s'inspire de l'intrigue du film en prises de vues réelles Le Voyage spatial, à la fois en termes d'histoire (un garçon qui se cache dans un vaisseau spatial) et de dessin (la rampe de lancement constructiviste incurvée, la surface de la lune, l'apesanteur et les mouvements des personnages). Ce premier film animé sur l'espace prolonge les narrations des années 1930 tout en mettant les enfants au premier plan du voyage spatial.

On trouve une autre interprétation dans Ott dans l'espace [Ott' kosmoses] (1961), réalisé par le réalisateur estonien Elbert Touganov après les premiers vols réussis dans l'espace: le voyage spatial n'est plus un rêve, c'est une expérience, un essai, 
un test. Le film est un film de poupées coloré et met en scène un petit garçon, Ott, peu attentif à l'école, qui se retrouve dans un camion. Il en descend près d'une rampe de lancement de fusées et monte dans une fusée qui décolle. Du fait de son manque d'enthousiasme pour apprendre et écouter, il voyage dans diverses planètes au lieu de rentrer directement sur la terre comme l'autopilote le lui commande. Il visite le Sahara où il se bat avec un lion ; la planète Neptune où il visite un royaume sous-marin; Mars, où il rencontre un dieu guerrier. Ces mondes sont représentés comme dans un film folklorique ou un conte, avec des héros qui rappellent les personnages des films d'Aleksandr Ptouchko des années 1940 et 1950. Finalement, Ott pousse accidentellement le bouton du voyage vers la lune, bien qu'il n'ait pas assez de combustible. Il se met à pleurer, réalisant qu'il a perdu son chemin et qu'il est incapable de s'appuyer sur la connaissance des images de ces planètes, qu'il aurait dû apprendre à l'école. Il est intéressant de noter qu'il comprend et suit les instructions de l'autopilote en estonien et que c'est son manuel de géographie en estonien qui le sauve, lui faisant comprendre quel bouton lui permettra de revenir sur terre, alors qu'il ne comprend pas les instructions en russe, écrites ou parlées. Il y a ici plus qu'un message sur l'utilité de l'école pour conquérir l'espace : seul l'estonien est capable d'enseigner des choses au garçon tandis que le russe (la langue du colonisateur) n'a aucun effet. Dans ce sens, l'exercice d'apprendre des choses sur l'espace (que l'on peut trouver également, par exemple, dans Mourzilka dans le spoutnik [Murzilka na Sputnike] (1960) de Evgueni Raïkovski et Boris Stepantsev) va au-delà de la dimension pédagogique du film, vers une individualisation de groupes ethniques et nationaux : il y a un groupe soviétique dans Le Vol pour la lune, mais l'identité nationale est déjà distincte dans les années 1960. Dans les films de la guerre froide, l'espace est un objet d'intérêt pour les enfants, et rien de sérieux n'est dit à propos des progrès techniques.

L'animation soviétique s'est moins intéressée à l'espace pendant les années de stagnation. Le thème est revenu dans le film de Roman Katchanov Le Mystère de la troisième planète [Tajna tret'ej planety] (1981), qui est l'une des nombreuses adaptations de l'écrivain de science-fiction Kir Boulytchev (1934-2003). Le film introduit à l'écran Alissa Selezneva, qui fera une nouvelle apparition en 2000 dans L'Anniversaire d'Alissa [Den' roždenija Alisy], que l'on étudiera infra. L'opposition entre le bien et le mal ne se situe pas ici entre la terre et les autres planètes, mais entre des bons et des méchants, comme dans le cinéma classique d'Hollywood. Réalisé à la fin de la stagnation, quand la course à la conquête de la lune avait été définitivement perdue au profit des Américains, le film met en scène Alissa, élève de CE2, et son père, le professeur Seleznev, dans un voyage spatial. Ils ne voyagent pas vers une planète définie mais sautent sans but d'une planète à l'autre, ils y rencontrent des humanoïdes bienveillants ou des robots entretenus par des 
SLOVO

190 À l'Est de Pixar : le film d'animation russe et soviétique - no 48/49

humains. Le film (qui vient d'être restauré par le Gosfilmofond ${ }^{6}$ ) a des couleurs très vives, les savants de l'année 2060 ressemblent aux hipsters des années 1950 devenus les hippies des années 1960 : ils portent les cheveux longs, des vêtements de couleurs vives et des lunettes carrées démodées, marchant (en apesanteur ?) à la manière des hipsters, balançant leurs hanches comme s'ils suivaient un rythme musical. Il y a des animaux de toutes sortes qui peuplent ces planètes et sont très aimés du savant. Les stations spatiales ressemblent à un intérieur domestique : elles sont recouvertes de tuiles, selon le design des années 1960. Les vaisseaux spatiaux ont des formes variées, ont des hublots en œil-de-bœuf comme dans les navires, et leur intérieur ressemble à un salon banal, avec des tasses, des soucoupes, une nappe. L'espace est comme une seconde maison et il est peuplé d'hommes (des pères, des savants) et d'enfants mais pas de femmes. Il n'y a qu'une grand-maman (sur terre) qui envoie un gâteau à son petit-fils et ses amis. Dans les films des années 1970, l'espace est un territoire masculin et un terrain de jeu pour les enfants, y compris dans des films en prises de vues réelles comme Moscou-Cassiopée [Moskva-Kassiopeja] (1973) et Des Adolescents dans l'univers [Otroki vo Vselennoj] (1974) de Richard Viktorov. Il est significatif que les explorateurs soient des adolescents et des enfants, et que les animaux domestiques jouent également un rôle primordial à l'exploration de l'espace : l'espace est une aire de jeux pour eux, répétant le confort domestique et constituant un miroir de la terre avec en plus divers attributs techniques (robots, machines, intérieurs au design géométrique); il y a toujours un lien stable avec la terre, ce qui la positionne comme le centre de l'univers.

\section{Les enfants dans l'espace}

La popularité en Russie du petit homme Neznaika [Neznajka: littéralement celui qui ne sait pas] est presque équivalente à celle de la poupée en peluche Tchébourachka, mais pas grâce aux films. Ces derniers, adaptés d'une série d'histoires écrites par le célèbre auteur pour enfants Nikolai Nossov (1908-1976), n'ont fait que renforcer la popularité du héros des livres. Le personnage de Neznaïka apparaît pour la première fois dans les comic strips de l'auteur et illustrateur canadien Palmer Cox (1840-1924) sur les Brownies ${ }^{7}$, des créatures fantastiques de sexe masculin, espiègles mais amusantes, qui ont servi de source d'inspiration pour la création des histoires de Nossov : Les Aventures de Neznaïka

6. Les archives nationales du cinéma (n.d.t.).

7. Traduits en russe en 1889 par Anna Xvol'son sous le titre Le Royaume des tout-petits [carstvo maljutok] et réédités plusieurs fois avant la révolution, mais pas après. 
et de ses camarades [Priključenija Neznajki i ego tovariščej] (1953-1954), avec le sous-titre «Une histoire conte de fées », suivi de Neznaïka dans la ville ensoleillée [Neznajka v solnećnom gorode] (1958) et du troisième volet de la trilogie, le «Roman conte de fées » Neznaïka sur la lune [Neznajka na lune] (1964).

La différence principale d'avec Palmer est que Nossov place l'action non pas dans un village d'elfes et de lutins, mais dans une commune d'enfants (par analogie avec des institutions comme le camp de pionniers ou l'orphelinat) pour garçons et filles, ce qui donne tout de suite l'occasion d'une série complémentaire d'intrigues et incarne les ambitions colonisatrices de l'URSS. Les petites créatures, ou les « courts-sur-pattes » [korotyški] ne sont pas des gnomes ni des lilliputiens, mais une curieuse création du point de vue anthropologique: ils acquièrent de l'expérience mais ne vieillissent pas, ils n'ont pas de pouvoirs magiques et n'ont pas de parents. Le héros a toutes les caractéristiques d'un enfant, mais ce n'est qu'un ersatz d'enfant. Les courts-sur-pattes sont infantiles et asexués, mais ils se différencient néanmoins selon leur sexe. On peut paradoxalement trouver un parallèle avec la culture populaire soviétique pourvoyeuse d'idéologie et créatrice du mythe soviétique. Neznaïka et ses amis existent dans un espace-temps mythologique qui confirme la distribution des savoir-faire: les fonctions des personnages sont données dans leur nom (Vintik: la vis, Tioubik: le tube), ce qui permet de les identifier comme des variations de héros culturels. Chaque personnage fonctionne comme un modèle de comportement, ce qui s'exprime par le biais de leur profession : le musicien Gouslia (les gusli), le poète Tsvetik (la petite fleur), le docteur Pilioulkin (du mot pilule), l'inventeur Znaika (qui sait). Cependant, ces rôles sont réservés aux personnages masculins, tandis que les filles s'occupent des corvées domestiques et de leur apparence, ce qui fait que la division par genre correspond aux stéréotypes traditionnels. Mais ils n'ont pas de vie sexuelle et présentent au jeune lecteur/spectateur le monde stérile de l'État soviétique idéal. Les courts-sur-pattes sont représentatifs : ce sont des types qui servent le régime dans sa tâche de colonisation, de préparation contre l'ennemi et de création de mécanismes de défense. Les illustrations d'Alekseï Laptev (1905-1965) et, après sa mort, de Genrikh Valk (1918-1998) ont joué un rôle essentiel dans la formation de l'image visuelle de Neznaika, le gentil enfant. Mais les attributs de Neznaika - en particulier, son habillement consistant en un chapeau bleu aux larges bords, un pantalon jaune canari et une chemise orange avec une cravate verte - sont ceux d'un adulte et sont typiques de la subculture des dandys et hipsters (les stiljagi), qui admiraient dans les années 1950 la mode et le mode de vie occidentaux. Neznaika incarne l'esprit rebelle des années 1960 : un voyou, un trickster, qui ne connaît aucune limite et rejette les normes de comportement établies. 
SLOVO

192 À l'Est de Pixar : le film d'animation russe et soviétique - no 48/49

En 1972 a été produite une mini-série de dix épisodes pour la télévision, dans laquelle le réalisateur suivait à la lettre l'histoire du premier livre et utilisait des poupées aux mouvements raides. Cinq ans plus tard, la deuxième histoire, Neznaïka dans la ville ensoleillée, a aussi été adaptée en dix épisodes. Le pays fantastique y est construit selon les principes du « socialisme développé » avec un niveau de progrès technique improbable, bien que les robots accomplissant les corvées domestiques aient été supprimés. La merveilleuse cité où arrive Neznaïka ressemble aux États satellites de l'URSS. Dans la série plus tardive de six épisodes, Neznaïka sur la lune [Neznajka na lune] (1997-2000), Neznaïka apparait comme un missionnaire. Le motif de la colonisation est présent ici aussi, mais, surtout, les effets comiques des explorations humaines dans l'espace tels que dans Le Voyage vers la lune de Méliès, mentionné supra, et son remake en 1908, Excursion dans la lune de Segundo de Chomón, sont amplifiés : les chercheurs rencontrent des autochtones, d'étranges humanoïdes, ils se présentent devant le monarque de la lune et sont chassés par la population locale pour avoir tenté de renverser la monarchie. Dans la version de Nossov, l'idée de renverser la structure bourgeoise se réalise sur la lune. Le groupe de courts-sur-pattes s'envole vers la lune, essaie d'établir le contact avec la population locale et combat le régime politique. Les courts-sur-pattes construisent une fusée et envoient une expédition dans la lune pour vérifier l'hypothèse de l'existence de leurs semblables sur la lune. Donc, quarante-huit petits hommes retournent sur le satellite de la terre. Le modèle de l'agression est une parodie d'une expédition militaire et de l'idée d'établir le contrôle sur un territoire. Neznaïka est en fait très peu agressif et il atterrit sur la lune par hasard, par erreur, car il a démarré la fusée et laissé les autres membres de l'expédition. Il vit de nombreuses aventures, jouant le rôle d'un businessman, d'un prolétarien et d'un clochard jusqu'à ce que les autres arrivent sur la lune pour amener la révolution sociale. L'animation, dessinée, est très proche esthétiquement de Disney et des films soviétiques des années 1960. Plusieurs personnages ont des voix qui rappellent celles de Balthazar Picsou, Mamie Baba, Flagada Jones et Archibald Picsou - les héros de la série La Bande à Picsou. Le retour à l'écran de Neznaikka, ce personnage culte de la littérature pour enfants de l'Union soviétique est aussi le symptôme d'un retour à l'idéologie correspondante.

La première version post-soviétique à l'écran des aventures d'Alissa Selezneva, l'hérö̈ne de Kir Boulytchev, est L'Anniversaire d'Alissa (2009) de Sergueï Sereguine. Le réalisateur s'est appuyé sur la tradition de l'animation russe avec un développement lent de l'action, ce qui a posé un problème avec le public ciblé. Il a pratiquement copié le film de 1981, Le Mystère de la troisième planète, l'héroïne manque de tempérament, de vivacité, de plasticité. Cependant, le film est une rare exception dans les œuvres qui ont pour thème l'espace parce que les longs-métrages tournent vite au mélodrame, ce qui se confirme dans les films avec Belka et Strelka, 
où la chienne cosmonaute sert sa patrie, protège sa famille et sa maison d'un ennemi extérieur. L'espace, selon l'idéologie de ces œuvres, est le territoire de la famille et pas du tout un endroit où l'on découvre et apprend de nouvelles choses, comme cela était le cas dans l'animation soviétique. Les héros du film vont rarement plus loin que la lune. Une lecture aussi « familiale et domestique » du thème du voyage spatial s'applique aussi aux films basés sur des motifs folkloriques.

Ivan Tsarévitch et le loup gris 2 [Ivan Carevič i seryj volk 2] (2013) de Vladimir Toropchine est la suite de l'un des gros succès produits par le studio de Saint-Pétersbourg Melnitsa [le moulin] en 2011. Ce film est construit sur une narration mélodramatique sans surprises; la lune et la terre correspondent aux représentations antiques (la terre est soutenue par trois éléphants debout sur une tortue géante) tandis que la vie domestique des héros est plutôt moderne. Un an a passé depuis le mariage d'Ivan Tsarévitch et de Vassilissa, qui est diplômée de la Sorbonne et d'Oxford, mais la tsarevna s'ennuie déjà des aventures romantiques. Elle n'est pas pressée de donner des petits-fils au tsar et rêve au lieu de cela de grands bals pour établir des liens avec les empires voisins. Ivan l'appelle « une jeune gâtée » et au lieu de mener une politique étrangère, il continue de renforcer les frontières de son 3 fois $9^{\mathrm{e}}$ royaume ${ }^{8}$. Et comme dans La Nuit de Noël [Noč pod roždestvom] (1913) de Ladislas Starewitch, où le forgeron Vakoula doit s'envoler pour Saint-Pétersbourg dans le but de voler les chaussures de la tsarine pour impressionner son Oksana bien-aimée, ici le père et le loup apprivoisé (l'ami d'Ivan et son auxiliaire magique) emploient l'acteur provincial Tchernomor pour mettre en scène l'enlèvement de la tsarevna et apporter un peu de fraîcheur dans la vie des jeunes mariés, ce qui s'achève pour le tsarévitch par un voyage dans la lune.

Tout ce qui a rapport au vol est fait avec beaucoup d'humour: le voyage du tsarévitch et du loup sur un boulet de canon entre en résonance avec Les Aventures de Munchbausen [Prikljućenija Mjunxauzena] (1973) et La Nuit de Noël-le loup fait le voyage sur le dos du tsarévitch comme Vakoula avec le diable, le tout au son de la sonate au clair de lune de Beethoven (mix) et de la voix mixée de Iouri Gagarine à la radio assourdie par un bruit de roues de train. Pendant le vol, le loup se comporte comme dans un voyage en train : il mange du poulet et des œufs durs en buvant $\mathrm{du}$ thé, fait des mots croisés. Le voyage vers la lune est représenté comme un voyage d'affaires. La fusée du retour vers la terre est particulièrement amusante: elle ressemble à une hutte avec des jambes humaines (dans les contes russes la maison de la sorcière Baba-Yaga a des pattes de poule) avec des ailes de poulet et des fenêtres

8. Le 3 fois $9^{\mathrm{e}}$ royaume dans les contes de fées russes est le royaume lointain que le héros atteint au bout de sa quête. (n.d.t.) 
SLOVO

194 À l'Est de Pixar : le film d'animation russe et soviétique - no 48/49

en bois décoré. Le séjour sur la planète déserte lui-même, cependant, est la partie la plus faible du film, réalisée avec l'esthétique de l'animation des années 1990, où l'atmosphère de vaudeville est plus importante que les effets spéciaux étourdissants.

Belka et Strelka 2. Aventures lunaires [Belka i Strelka 2. Lunnyepriključenija, 3D] (2014) d'Aleksandr Khramtsov, Inna Evlakinnova et Vadim Sotskov, la suite de Belka et Strelka, les Chiens des étoiles [Belka i Strelka, Zvëzdnye sobaki] (2010) de Sviatoslav Ouchakov et Inna Evlannikova, ressemble à un puzzle fabriqué de clichés et de truismes. La narration se développe dans la tradition des films de la guerre froide, à la différence près que les films soviétiques écartaient de l'écran tout contact entre les citoyens soviétiques et les créatures extraterrestres. Ici, nous avons un représentant d'un type nouveau : la poupée Pouchok, un chiot très sociable. Le fils de la célèbre chienne astronaute Belka et de son mari, l'entraîneur d'astronautes Kazbek, a grandi dans la maison du président américain, mais son amour pour la patrie des ingénieurs Konstantin Tselkovski et Sergueï Korolev, ainsi que de Gagarine l'a nourri pendant toute son enfance. Il a la nature héroïque de son père plutôt brutal et le cœur tendre de sa mère, de qui il a hérité la couleur de son pelage.

Pendant que Strelka est occupée égoïstement à chercher sa valise d'os, Belka - la loyauté même - cherche à aider sa famille. Pouchok est entouré d'attentions et de soins, il est le favori de la fille du président et provoque l'envie des autres animaux de la maisonnée. Le chiot a entendu parler de ses parents biologiques et rêve d'aller dans l'espace.

La réalité créée dans le film est très pro-soviétique. Les chiens russes partent pour un tour du monde pendant que les singes envoyés dans l'espace par les USA ont échoué dans leur mission et sont couchés dans des lits d'hôpital. Les dialogues contiennent des références à des histoires drôles, et les héros nationaux sont étroitement liés à des animaux : pour les Américains, c'est le chimpanzé, pour les Juifs, c'est le rat plein de ressources Vénia, et pour les Russes, des chiens de toutes les couleurs. Le film est saturé de citations connues de films soviétiques et de parodies de Brejnev, et ses dialogues s'adressent à la vieille génération, les enfants ne peuvent être amusés par ces dialogues et ces gags.

Peu à peu, les gentils chiens se transforment en personnages-types déplaisants qui exploitent les clichés véhiculés par les histoires drôles. Après leur visite, la lune devient un parc d'attractions, avec la statue de la liberté, la tour Eiffel et d'autres monuments de la civilisation terrienne éclairés par des néons, et tout cela ne fera certainement pas sourire les adultes ou réagir les enfants. Les héros quittent la lune et reviennent travailler et étudier, car la vie normale sur une autre planète est impossible. Cependant, ils ne peuvent pas non plus remettre la lune dans son état originel, les auteurs confient cela à des représentants humanoïdes d'une autre galaxie. 
Belka et Strelka est politiquement correct et offre une véritable réflexion sur la situation socioculturelle : après les aventures sur la lune, la famille canine se disperse dans tout le globe terrestre avec la condition qu'ils se retrouveront en URSS pendant les vacances. Les astronautes russes préferent donner à leurs enfants une éducation en Occident. Les normes de genre reflétées dans le titre soulignent la supériorité féminine, cependant, les héros sont des mâles: Kazbek et Pouchok dans Aventures lunaires. C'est un fait historique que le président américain a reçu une « fille », mais les auteurs de ce biopic des chiens astronautes ont changé cela, insistant sur le fait que c'est un chien mâle, Pouchok, qui défend l'honneur de la patrie. De plus, à la fin, un modèle de famille est annoncé pour Pouchok : l'élégante Fifi, qui ressemble à sa mère, va tomber amoureuse du gracieux fils de Belka dans un pays étranger. Il y aura donc peut-être une suite.

Le remake animé du film satirique Kin-Dza-Dza (1985) du patriarche de la comédie russe, Gueorgui Danielia, Kou! Kin-Dza-Dza (2013), réalisé également par lui en collaboration avec Tatiana Ilina, commence au début du XXI ${ }^{\mathrm{e}}$ siècle dans une Moscou hivernale. Un violoncelliste mondialement connu, Vladimir Tchijov, dit l'oncle Vova, rentre chez lui après un concert en solo au conservatoire. Dans le nouveau quartier de Moscou-City, son véhicule est coincé dans un embouteillage. Ne souhaitant pas attendre, il sort de la voiture, continue à pied et rencontre un adolescent, Tolik Tsarapkine, qui se présente comme son neveu. À ce moment-là, un homme pieds nus avec une couronne de fleurs de camomille court à leur rencontre, tenant un appareil qui a des touches comme un téléphone portable, et prononce la phrase fatale, axiomatique: «Dites-moi le numéro de votre galaxie. Sur quel bouton dois-je appuyer pour rentrer chez moi ? ». Prenant le passant pour un drogué ou un fou, Tolik lui dit d'appuyer sur 03, le numéro des urgences médicales. L'étranger rétorque: «03, c'est Pliouk, là où habitent les Tchatlanians ». Mais Tolik ne l'écoute pas, appuie sur le numéro, et Tchijov et lui se retrouvent soudain dans un désert avec deux soleils. Deux peuples habitent sur la planète Pliouk : les Tchatlanians, qui dominent, et les Patsaks, qui sont dominés. À leur arrivée, les héros rencontrent un trio pittoresque: Uef le Tchtatlanien, Bi le Patsak et le robot-taupe Abradoks, qui voyagent dans un appareil volant, le « pepelats ». Un dictateur, P.J., règne sur cet univers désertique. Les extra-terrestres sont des humanoïdes, à l'exception d'Abradoks, et l'animation souligne et renforce le grotesque de leur physique. Ils se parlent avec un vocabulaire limité à deux mots, kou et kiou, sorte d'euphémismes qui remplacent toutes sortes de mots. La hiérarchie entre les individus se fait en fonction de la quantité de monnaie d'échange que l'on possède : sur cette planète, tout se paye, cet univers fonctionne donc sur un modèle capitaliste. Les deux terriens reçoivent le statut de Patsaks, donc de créatures inférieures, ce qui les oblige à porter une cloche spéciale (le «tsak ») dans le nez; quand ils rencontrent un Tchatlanian, ils doivent effectuer un rituel 
SLOVO

196 À l'Est de Pixar : le film d'animation russe et soviétique - no 48/49

de courtoisie qui consiste à se frapper les deux joues. Obtenir le «gravitsapa», l'appareil qui permettrait au «pepelats » de bouger vers une autre planète, n'est pas une tâche facile. Dans leurs efforts pour rentrer sur terre, ils rencontrent des voleurs, font face plusieurs fois à la mort, se retrouvent en prison et même dans la résidence du gouverneur, et finissent par atterrir sur une planète déserte où l'oxygène manque et où la tentation de se laisser mourir s'empare des deux héros, désespérés de ne pouvoir rentrer sur terre. À ce moment-là, apparaît l'homme pieds-nus à la couronne de fleurs de camomille, qui les renvoie sur terre. Le début de la première scène du film se répète, mais tout est rentré dans l'ordre, la structure circulaire semble effacer le séjour sur Pliouk, et seul le réflexe qu'ont les deux héros de faire le rituel du salut patsak au passage d'un chasse-neige témoigne de ce qu'ils ont bien vécu cette aventure.

Le film d'animation exige de la contemplation, un mode spécifique de perception et laisse une part au non exprimé, fonctionnant comme une composition musicale. L'usurpateur P. J. donne le ton des concerts et est le chef censeur. Le robot Abradoks, pragmatique et sans scrupules, rêve de faire une carrière solo. Le propriétaire d'un manège accepte de faire traverser un abîme à l'oncle Vova, qui a le vertige, contre paiement mais le regrette amèrement ensuite car il est obligé de l'écouter réciter désespérément le poème «Le pétrel » de Maksim Gorki. Des musiciens ambulants sont prêts à accueillir les terriens sur leur « automobile » du moment que leur sécurité n'est pas menacée. En plus d'un langage différent et de son vocabulaire propre, bien que limité, le film promeut un nouveau paysage sonore. Quand les habitants de la planète meurent, ils laissent derrière eux leur respiration, comme un mot non dit ou un son inarticulé. Il y a plus de tombes dans le film animé que dans le film de 1985 et elles constituent un élément important du paysage : en forme de ballons, elles sont les exhalations des personnes mortes sous un régime autoritaire.

Les réalisateurs concentrent leur attention non pas sur les êtres humains, mais sur les humanoïdes de la planète. De ce fait, on peut définir le genre comme une fantasmagorie plutôt qu'une satire. En tant que remake du film de la perestroikka, ce film explore l'espace comme une anti-utopie : ce n'est pas un endroit où les terriens voudraient vivre. Les héros ne rencontrent aucune empathie ni aucune curiosité chez les extra-terrestres, mais seulement de l'agressivité, de la malhonnêteté et de la cupidité. Dans sa critique du film, Sasha Senderovich a étudié en détail la signification allégorique du film et a conclu que le film est une allégorie de ladivision sociale de la Russie : « la planète Pliouk n'est pas vraiment différente de la Russie de Poutine9 ». 


\section{Conclusion}

Dans le cinéma d'animation russe et soviétique, les autres planètes ressemblent à la terre et semblent en dire plus sur notre propre planète que sur l'espace et la vie extra-terrestre. Si le voyage et l'exploration eux-mêmes ainsi que le désir de découvrir dominaient dans l'animation soviétique sur l'espace, l'animation post-soviétique retourne vers les intrigues de la science-fiction et vers les mythes soviétiques sur l'espace, mais les héros de ces mythes du passé ont changé : l'espace est peuplé d'enfants et d'animaux (principalement des chiens) et il se transforme en terrain de jeu, ce qui le rend tout à fait inintéressant en tant que domaine de pouvoir politique. Les autres planètes servent de miroirs dans lequel les personnages reflètent la vie d'aujourd'hui sur terre. L'espace est devenu une part de la normalité et n'est plus un objet de conquête ni de compétition avec l'Amérique : le temps de la course à l'espace n'est plus, et les voyages conjoints dans l'espace et les stations spatiales habitées multinationales ont remplacé les discours défensifs de l'ère soviétique. Ce qui reste, c'est la domestication de l'espace, avec des enfants et des animaux, des plantes en pot et des oiseaux, et l'illusion que l'espace est un terrain de jeu alternatif.

\section{Bibliographie}

Beumers Birgit, 2017, "Imaginings of Space, or a Journey Through Special Effects", in Goscilo Helena \& STrukov Vlad (ed.), Russian Aviation, Space Flight and Visual Culture, Routledge, Londres, pp. 169-188.

Klušancev Pavel, 1997, Odna iz moix kartin. Zapiski kinorežisëra (Doroga $k$ zvëzdam), [Un de mes films. Notes d'un réalisateur (La voie des étoiles)], Goskino Rossii et Sankt-Peterburgskij Institut Kino i Televidenija, Saint-Pétersbourg.

Senderovich Sasha, 2014, «Critique de "Ku! Kin-dza-dza” » in Kinokultura $\mathrm{n}^{\circ}$ 43, http://www.kinokultura.com/2014/43r-ku-kindzadza.shtml, consulté en décembre 2017.

Siddier Asif, 2008, «Imagining the Cosmos: Utopians, Mystics, and the Popular Culture of Spaceflight in Revolutionary Russia », in Osiris, $n^{\circ} 23$, The University of Chicago Press, Chicago, pp. 260-288. 
SLOVO

198 À l'Est de Pixar : le film d'animation russe et soviétique - no 48/49

Sputnitskaia Nina, 2017, «The Politics of Outer Space: Colonisers and Missionaries in Russian Fantasy Film of the 1930s », in Studies in Russian and Soviet Cinema, n 11, vol. 2, pp. 134-145.

\section{Filmographie}

BRUMBerg Valentina et Zinaida, 1953, Polët na lunu [vol pour la lune], Soïouzmoultfilm.

Butyrin Jurij, Ignatenko Andrej \& Ljutkevič Aleksandr, 1997-2000, Neznajka na lune [Neznaika sur la lune], Studio FAF et compagnie, Rousskoïe zoloto.

Danielija Georgij, 1985, Kin-Dza-Dza, Moscou, Mosfilm.

Danielija Georgij \& IL'Ina Tat'jana, 2012, Kou! Kin-Dza-Dza, Moscou, STV, Ritm, Iougra-films.

Evlakinnova Inna \& Ušakov Svjatoslav, 2010, Belka i Strelka, Zvëzdnye sobaki [Belka et Strelka. Les chiens des étoiles], Moscou, Tsentr natsionalnogo filma.

Evlakinnova Inna, Xramcov Aleksandr \& Sockov Vadim, 2014, Belka i Strelka 2. Lunnye prikljucenija [Belka et Strelka 2. Aventures lunaires], 3D, Moscou, Tsentr natsionalnogo filma.

Katchanov Roman, 1981, Tajna tret'ej planety [Le mystère de la troisième planète], Moscou, Soïouzmoultfilm.

Khodataev, Nikolaï, Komissarenko Zenon \& Merkoulov Iouri, 1924, Mežplanetnaja revoljucija [La révolution interplanétaire], Moscou, Mejrabprom-Rous et GTK.

KLušancev Pavel, 1961, Planeta bur' [La planète des tempêtes], Moscou, Soïouzmoultfilm.

Klušancev, Pavel, 1965, Luna [La lune], Moscou, Soïouzmoultfilm.

Klušancev, Pavel, 1968, Mars, Moscou, Soïouzmoultfilm. 
Protazanov Jakov, 1924, Aèlita [Aelita], Moscou, Mejrabprom-Rous.

Rajkovskij Evgenij \& Stepancev Boris, 1960, Murzilka na Sputnike [Mourzilka dans le spoutnik], Moscou, Soïouzmoultfilm.

Sereguine Sergueï, 2009, Den' roždenija Alisy [L'anniversaire d'Alissa], Moscou, Masterfilm.

Toropchine Vladimir, 2013, Ivan Carevič i seryj volk 2 [Ivan Tsarévitch et le loup gris 2], Saint-Pétersbourg, Melnitsa et STV.

Touganov Èl'bert, 1961, Ott' kosmoses [Ott dans l'espace], Tallin, studios Tallinfilm.

Viktorov Richard, 1973, Moskva-Kassiopeja [Moscou-Cassiopée], Moscou, studio Gorki.

Viktorov Richard, 1974, Otroki vo Vselennoj [Des adolescents dans l'univers], Moscou, studio Gorki.

Žuravlev Vasilij, 1935, Kosmičeskij rejs [Le vol spatial], Moscou, Mosfilm.

Résumé : Cet article montre qu'avec le passage de l'ère soviétique à l'ère post-soviétique, l'espace dans le film d'animation s'est transformé d'un territoire nouveau de conquête et d'exploration en un miroir négatif de la terre, que l'on est passé de l'utopie à la dystopie et que l'espace n'est plus un endroit désirable pour être habité, ce qui sous-entend que c'est la terre elle-même qui est devenue indésirable. Avec l'utilisation de l'animation pour créer des effets nécessaires à la représentation réaliste (dans les œuvres d'Aleksandr Ptouchko et Pavel Klouchantsev, par exemple), l'espace a servi d'alternative parfaite à la réalisation du style de vie soviétique. Cependant, après que les Américains ont mis le pied sur la lune, il s'est produit un décalage, et les autres planètes sont devenues des répliques bourgeoises et domestiques, l'équivalent de l'utopie soviétique du «socialisme développé ». La colonisation n'a pas une signification politique, elle offre des espaces de vie alternatifs pour des familles avec enfants et animaux domestiques. Le voyage dans la lune est une expérience et un test de maturité, dans lequel la conquête n'est plus d'actualité, l'espace est un terrain de jeux pour enfants et adolescents, il procure du confort domestique et sert de miroir réfléchissant de la terre avec quelques attributs techniques; le lien avec la terre est conservé, la terre étant toujours le centre de 
SLOVO

200 À l'Est de Pixar : le film d'animation russe et soviétique - no 48/49

l'univers. Dans le nouveau millénaire, les remake et suites de films montrent l'infantilisation des exploits cosmiques (avec la figure de Neznaika/Jenesaispas) et dressent le portrait d'adolescents en hipsters révoltés, tandis que Ku! Kin-Dza-Dza décrit l'autre planète comme un espace dystopique, indésirable pour l'intelligentsia créative.

Mots-clefs : animation, espace, colonisation, exploration, dystopie, utopie.

Abstract: This article argues that, over the Soviet and into the post-Soviet era, outer space in animated films has turned from a new, unknown territory for conquest and exploration into an undesirable mirror of earth, from a utopia into a dystopia, a space that is no longer desirable for habitation, inferring that it is earth itself which has become undesirable. Following the use of animation to create the effects necessary for a realistic representation of spaceflight (e.g. in the works of Aleksandr Ptushko and Pavel Klushantsev), outer space served as a perfect alternative for the realisation of a socialist lifestyle. However, after the American landing on the moon a shift takes place, which turns other planets into domestic and bourgeois little replica worlds, the equivalent of Soviet utopia under "developed socialism". Colonization has less a political significance as one of finding alternative living spaces for families, with children and pets. Space travel is an experience and a test of maturity, where conquest no longer relevant and outer space is a playground for children and teenagers, providing domestic comfort and serving as a mirror reflection of earth with some technical attributes, whilst always maintaining a link with earth, which remains at the centre of the universe. In the new millennium animated remakes and sequels show the infantilization of cosmic exploits (in the figure of Neznaika, or Dunno), portraying teenagers as rebellious hipsters, while $\mathrm{Ku}$ ! Kin-Dza-Dza portrays the other planet as a dystopian space, undesirable for the creative intelligentsia.

Keywords: animation, space, colonization, exploration, dystopia, utopy.

Абстракт: B статье доказьвается, что в советских и постсоветских мультипликачионньх фильмах космическое пространство постепенно превратилось из утопического пространства в антиутопическое, из таинственной, привлекательной для завоевания и исследования территории - в нелицеприятную копию Земли, пространство непригодное для обитания. Благодаря опьтамиспользования анимачиидлясозданияэффектов, позволивших достоверно представить космический полет и другие планеть (например, в работах Александра Птушко и Павла Клушаниева), космос предстал прекрасной площадкой для реализачии идей сочиалистического строительства. Однако после высадки американцев на Ауну происходит пересмотр сюжетов и мотивировок, в духе советской утопии «развитого сочиализма» другие 
планеты интерпретируются как внутренние и буржуазнье маленькие копии миров. Постепенно утрачивается политическое значение завоевания космоса, идея колонизации вытесняется поиском альтернативных мест проживания для семей с детьми и домашними животньми. Космические путешествия это опьт и иничиачия, но открытие космических глубин уже не актуально, инопланетное пространство служит для детей и подростков своего рода игровой пломадкой, обеспечиваюочей домашний $к$ омфорт и служащей зеркальным отражением быта землян со всеми техническими атрибутами. При этом всегда поддерживается связь с Землей (сродителями), которая воспринимается как центр Вселенной. В новом тысячелетии анимационные ремейки и сиквельь демонстрируют инфантилизачию космических подвигов (например, в образе Незнайки), изображая подростков бунтуюшими хипстерами, в то же время Ку! Кин-Аза-Аза интерпретирует иную планету как антиутопическое пространство, в котором нет места творческой интеллигенции.

Ключевье слова: анимачия, космос, колонизауия, открьватели, дистопия, ymonus. 